\title{
On inversion of adjunction
}

\author{
By Osamu Fujino and Kenta Hashizume \\ Department of Mathematics, Graduate School of Science, Kyoto University, \\ Kitashirakawa Oiwake-cho, Sakyo-ku, Kyoto 606-8502, Japan \\ (Communicated by Shigefumi MoRI, M.J.A., Jan. 12, 2022)
}

\begin{abstract}
We first announce our recent result on adjunction and inversion of adjunction. Then we clarify the relationship between our inversion of adjunction and Hacon's inversion of adjunction for log canonical centers of arbitrary codimension.
\end{abstract}

Key words: Adjunction; inversion of adjunction; minimal model program.

1. Introduction. In [9], we established the following adjunction and inversion of adjunction for $\log$ canonical centers of arbitrary codimension in full generality.

Theorem 1.1 (Adjunction and Inversion of Adjunction, see [9]). Let $X$ be a normal variety and let $\Delta$ be an effective $\mathbf{R}$-divisor on $X$ such that $K_{X}+\Delta$ is $\mathbf{R}$-Cartier. Let $W$ be a log canonical center of $(X, \Delta)$ and let $\nu: Z \rightarrow W$ be the normalization of $W$. Then there exist a b-potentially nef $\mathbf{R}$-b-divisor $\mathbf{M}$ and an $\mathbf{R}$-b-divisor $\mathbf{B}$ on $Z$ such that $\mathbf{B}_{Z}$ is effective with

$$
\nu^{*}\left(K_{X}+\Delta\right)=\mathbf{K}_{Z}+\mathbf{M}_{Z}+\mathbf{B}_{Z} .
$$

More precisely, there exists a projective birational morphism p: $Z^{\prime} \rightarrow Z$ from a smooth quasi-projective variety $Z^{\prime}$ such that

(i) $\mathbf{M}=\overline{\mathbf{M}_{Z^{\prime}}}$ and $\mathbf{M}_{Z^{\prime}}$ is a potentially nef $\mathbf{R}$-divisor on $Z^{\prime}$,

(ii) $\mathbf{K}+\mathbf{B}=\overline{\mathbf{K}_{Z^{\prime}}+\mathbf{B}_{Z^{\prime}}}$,

(iii) Supp $\mathbf{B}_{Z^{\prime}}$ is a simple normal crossing divisor on $Z^{\prime}$,

(iv) $\nu \circ p\left(\mathbf{B}_{Z^{\prime}}^{>1}\right)=W \cap \operatorname{Nlc}(X, \Delta)$ holds set theoretically, and

(v) $\nu \circ p\left(\mathbf{B}_{\bar{Z}^{\prime}}^{>1}\right)=W \cap\left(\operatorname{Nlc}(X, \Delta) \cup \bigcup_{W \not W^{\dagger}} W^{\dagger}\right)$, where $W^{\dagger}$ runs over log canonical centers of $(X, \Delta)$ which do not contain $W$, holds set theoretically.

For the details of Theorem 1.1, see [9]. On the other hand, Hacon introduced a b-divisor, which is denoted by $\mathbf{B}(W ; X, \Delta)$, and formulated his inversion of adjunction for $\log$ canonical centers of arbitrary codimension in [10], and the b-divisor is

2010 Mathematics Subject Classification. Primary 14E30; Secondary $14 \mathrm{~N} 30$. also studied in the context of generalized pairs in $[3$, Theorem 6.7]. We note that the definition of $\mathbf{B}(W ; X, \Delta)$ is different from our definition of $\mathbf{B}$ in Theorem 1.1. The goal of this paper is to prove that $\mathbf{B}(W ; X, \Delta)=\mathbf{B}$ always holds. The following theorem is the main result of this paper.

Theorem 1.2. Let $X$ be a normal variety and let $\Delta$ be an effective $\mathbf{R}$-divisor on $X$ such that $K_{X}+\Delta$ is $\mathbf{R}$-Cartier. Let $W$ be a log canonical center of $(X, \Delta)$. Then Hacon's $\mathbf{B}(W ; X, \Delta)$ coincides with the $\mathbf{R}$-b-divisor $\mathbf{B}$ on $Z$ in Theorem 1.1, where $Z$ is the normalization of $W$. Hence our adjunction and inversion of adjunction for $\log$ canonical centers of arbitrary codimension completely generalizes Hacon's inversion of adjunction.

By Theorem 1.2, Hacon's inversion of adjunction for log canonical centers of arbitrary codimension in [10] now becomes a very special case of Theorem 1.1. We think that the definition of $\mathbf{B}$ in [8] and [9] is more natural than Hacon's definition of $\mathbf{B}(W ; X, \Delta)$ in [10]. However, $\mathbf{B}(W ; X, \Delta)$ seems to be easier to compute than $\mathbf{B}$. Hence Theorem 1.2 is important and useful.

Remark 1.3. In [10], Hacon defined $\mathbf{B}(W$; $X, \Delta)$ under the extra assumption that $\Delta$ is a boundary Q-divisor on $X$ such that $K_{X}+\Delta$ is Q-Cartier. However, his definition works for effective $\mathbf{R}$-divisors $\Delta$ such that $K_{X}+\Delta$ is $\mathbf{R}$-Cartier without any modifications. By definition, it is obvious that $\mathbf{B}(W ; X, \Delta) \leq \mathbf{B}$ always holds.

Let us quickly explain the proof of Theorem 1.1 for the reader's convenience. First we take a suitable resolution of singularities of the pair $(X, \Delta)$. Next, by using the framework of quasi-log schemes (see [6, Chapter 6]), we construct a natural quasi-log scheme structure on $Z$ (see [7]). Then we 
apply the theory of basic R-slc-trivial fibrations and obtain $\mathbf{B}$ and $\mathbf{M}$ satisfying (i), (ii), (iii), and (v) (see [9]). Finally, we prove (iv) with the aid of the minimal model program for log canonical pairs (see [8]). We strongly recommend the interested reader to see [7], [8], and [9].

In this paper, we will only use the minimal model program at the level of [2]. We will freely use the standard notation and definitions of the minimal model program as in [5] and [6] (see also [7]).

2. $\mathbf{B}(\boldsymbol{W} ; \boldsymbol{X}, \boldsymbol{\Delta})$ and $\mathbf{B}$. Let us recall the definition of $\mathbf{B}(W ; X, \Delta)$ and $\mathbf{B}$.

Definition $2.1(\mathbf{B}(W ; X, \Delta)$ and $\mathbf{B})$. Let $X$ be a normal variety and let $\Delta$ be an $\mathbf{R}$-divisor on $X$ such that $K_{X}+\Delta$ is $\mathbf{R}$-Cartier and that $\Delta$ is effective in a neighborhood of the generic point of a closed subvariety $W$. Assume that $W$ is a log canonical center of $(X, \Delta)$. Let $\nu: Z \rightarrow W$ be the normalization of $W$. For any proper birational morphism $\rho: \tilde{Z} \rightarrow Z$ from a normal variety $\tilde{Z}$, we consider prime divisors $T$ over $X$ such that $a(T, X, \Delta)=-1$ and that the center of $T$ on $X$ is $W$. We take a suitable resolution $f: Y \rightarrow X$ with $K_{Y}+\Delta_{Y}=f^{*}\left(K_{X}+\Delta\right)$ so that $\Delta_{Y}$ is a simple normal crossing divisor on $Y, T$ is a prime divisor on $Y$, and the induced map $f_{T}: T \rightarrow \tilde{Z}$ is a morphism. We put $\Delta_{T}=\left.\left(\Delta_{Y}-T\right)\right|_{T}$. For any prime divisor $P$ on $\tilde{Z}$, we shrink $\tilde{Z}$ and assume that $P$ is Cartier. Then we define a real number $\alpha_{P, T}$ by

$$
\alpha_{P, T}=\sup \left\{\begin{array}{l|l}
\lambda \in \mathbf{R} & \begin{array}{l}
\left(T, \Delta_{T}+\lambda f_{T}^{*} P\right) \text { is sub } \\
\text { log canonical over the } \\
\text { generic point of } P
\end{array}
\end{array}\right\} .
$$

It is easy to see that $\alpha_{P, T}$ is independent of the resolution $f: Y \rightarrow X$ and is well-defined. The trace $\mathbf{B}_{\tilde{Z}}$ of $\mathbf{B}$ on $\tilde{Z}$ is defined by

$$
\mathbf{B}_{\tilde{Z}}=\sum_{P}\left(1-\inf _{T} \alpha_{P, T}\right) P
$$

where $P$ runs over prime divisors on $\tilde{Z}$ and $T$ runs over prime divisors over $X$ such that $a(T, X, \Delta)=$ -1 and that the center of $T$ on $X$ is $W$.

We choose and fix one prime divisor $T$ over $X$ such that $a(T, X, \Delta)=-1$ and that the center of $T$ on $X$ is $W$. The trace $\mathbf{B}(W ; X, \Delta)_{\tilde{Z}}$ of $\mathbf{B}(W ; X, \Delta)$ is defined by

$$
\mathbf{B}(W ; X, \Delta)_{\tilde{Z}}=\sum_{P}\left(1-\alpha_{P, T}\right) P
$$

where $P$ runs over prime divisors on $\tilde{Z}$. By definition, $\mathbf{B}(W ; X, \Delta) \leq \mathbf{B}$ always holds.

Remark 2.2. Although it is not obvious, we can check that $\mathbf{B}$ is a well-defined $\mathbf{R}$-b-divisor on $Z$. On the other hand, we can easily see that $\mathbf{B}(W ; X, \Delta)$ is a well-defined $\mathbf{R}$-b-divisor on $Z$, but it is not clear whether $\mathbf{B}(W ; X, \Delta)$ is independent of the choice of $T$ or not. In Theorem 1.2, we prove that $\mathbf{B}=\mathbf{B}(W ; X, \Delta)$ holds. This implies that $\mathbf{B}(W ; X, \Delta)$ is independent of the choice of $T$. Moreover, by the proof of Theorem 1.2, the welldefinedness of $\mathbf{B}$ is clear.

Precisely speaking, Hacon claims that $\mathbf{B}(W ; X, \Delta)$ is independent of the choice of the divisor $T$ without proof in [10]. In this paper, we prove it in a slightly more general setting.

3. Proof of Theorem 1.2. In this section, we prove Theorem 1.2. Before the proof of Theorem 1.2 , we prepare three lemmas.

Lemma 3.1. Let $X$ be a normal variety and let $\Delta$ be an $\mathbf{R}$-divisor on $X$ such that $K_{X}+\Delta$ is $\mathbf{R}$-Cartier. Let $f: X \rightarrow Y$ be a projective surjective morphism onto a smooth curve $Y$ such that $K_{X}+\Delta \sim_{\mathbf{R}, f} 0$. Let $P$ be a closed point of $Y$ such that $(X, \Delta)$ is divisorial log terminal over $Y \backslash P$. We take the log canonical threshold $b_{P}$ of $(X, \Delta)$ with respect to $f^{*} P$. Let $F$ be a connected component of $f^{-1}(P)$. Assume that $F$ contains a $\log$ canonical center of $\left(X, \Delta+b_{P} f^{*} P\right)$. Let $S$ be an irreducible component of $\left(\Delta^{h}\right)^{=1}$, that is, $S$ is a codimension one log canonical center of $(X, \Delta)$ which is dominant onto $Y$ by $f$. Then $S \cap F$ always contains a log canonical center of $\left(X, \Delta+b_{P} f^{*} P\right)$. Hence, if $\nu: S^{\nu} \rightarrow S$ is the normalization of $S$ and $\Delta_{S^{\nu}}$ is the R-divisor on $S^{\nu}$ defined by $K_{S^{\nu}}+\Delta_{S^{\nu}}=\nu^{*}\left(K_{X}+\right.$ $\Delta)$, then $\left(S^{\nu}, \Delta_{S^{\nu}}+b_{P} \nu^{*}\left(\left.f\right|_{S}\right)^{*} P\right)$ has a log canonical center mapping to $P$.

Proof. Without loss of generality, we may assume that $Y$ is quasi-projective by shrinking $Y$ around $P$. By replacing $\Delta$ with $\Delta+m f^{*} P$ for some sufficiently large positive integer $m$, we may assume that $\Delta$ is effective with $\Delta \geq f^{*} P$. In this situation, the $\log$ canonical threshold $b_{P}$ is a nonpositive number. We take a resolution of singularities of $X$ suitably and run a minimal model program with scaling of an ample divisor as in the proof of [7, Theorem 3.9]. Then we have a dlt blow-up $g: Z \rightarrow X$ of $(X, \Delta)$ with $K_{Z}+\Delta_{Z}=g^{*}\left(K_{X}+\Delta\right)$ such that

- $Z$ is $\mathbf{Q}$-factorial, 
- $g$ is small over $Y \backslash P$, and

- the pair $\left(Z, \Delta_{Z}^{<1}+\operatorname{Supp} \Delta_{\bar{Z}}^{>1}\right)$ is divisorial $\log$ terminal.

For the details of the construction of $g: Z \rightarrow X$, see [7, Theorem 3.9]. By replacing $f:(X, \Delta) \rightarrow Y$ and $F$ with $f \circ g:\left(Z, \Delta_{Z}\right) \rightarrow Y$ and $g^{-1}(F)$ respectively, we may assume that $X$ is $\mathbf{Q}$-factorial and $\left(X, \Delta^{<1}+\right.$ $\left.\operatorname{Supp} \Delta^{\geq 1}\right)$ is divisorial $\log$ terminal. Hence $(X,(\Delta+$ $\left.b_{P} f^{*} P\right)^{>0}$ ) is a $\mathbf{Q}$-factorial divisorial $\log$ terminal pair. Note that

$$
K_{X}+\left(\Delta+b_{P} f^{*} P\right)^{>0} \sim_{\mathbf{R}, f}-\left(\Delta+b_{P} f^{*} P\right)^{<0} \geq 0 .
$$

If $\operatorname{Supp}\left(\Delta+b_{P} f^{*} P\right)^{=1} \supset F$, then it is obvious that $\Delta+b_{P} f^{*} P$ is effective in a neighborhood of $F$ and that $S \cap F$ contains a $\log$ canonical center of $\left(X, \Delta+b_{P} f^{*} P\right)$. Therefore, from now on, we assume that $\operatorname{Supp}\left(\Delta+b_{P} f^{*} P\right)^{=1} \not \supset F$. Let $F=\sum_{i} F_{i}$ be the irreducible decomposition of $F$. If $F_{i} \not \subset$ $\operatorname{Supp}\left(\Delta+b_{P} f^{*} P\right)^{=1}$, then we take $0<\varepsilon_{i} \ll 1$. If $F_{i} \subset \operatorname{Supp}\left(\Delta+b_{P} f^{*} P\right)^{=1}$, then we put $\varepsilon_{i}=0$. Let $f: X \stackrel{h}{\longrightarrow} \rightarrow Y$ be the Stein factorization. Then $\left(X,\left(\Delta+b_{P} f^{*} P\right)^{>0}+\sum_{i} \varepsilon_{i} F_{i}\right)$ is a $\mathbf{Q}$-factorial divisorial log terminal pair with

$$
\begin{aligned}
K_{X} & +\left(\Delta+b_{P} f^{*} P\right)^{>0}+\sum_{i} \varepsilon_{i} F_{i} \\
& \sim_{\mathbf{R}, h}-\left(\Delta+b_{P} f^{*} P\right)^{<0}+\sum_{i} \varepsilon_{i} F_{i} \geq 0 .
\end{aligned}
$$

We shrink $\bar{Y}$ around $h(F)$ and run a minimal model program of $K_{X}+\left(\Delta+b_{P} f^{*} P\right)^{>0}+\sum_{i} \varepsilon_{i} F_{i}$ over $\bar{Y}$ with scaling of an ample divisor. Then, after finitely many steps, we get $X^{\prime}$ with the following commutative diagram:

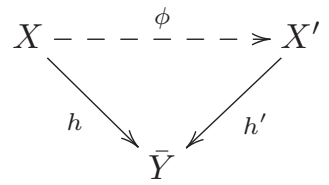

such that $\Delta^{\prime}+b_{P}\left(f^{\prime}\right)^{*} P$ is effective in a neighborhood of $F^{\prime}$ and that $\left(\Delta^{\prime}+b_{P}\left(f^{\prime}\right)^{*} P\right)^{=1} \geq F^{\prime}$, where $f^{\prime}: X^{\prime} \rightarrow \bar{Y}, \Delta^{\prime}=\phi_{*} \Delta$, and $F^{\prime}=\phi_{*} F$. For the details of the above minimal model program, see [4] (see also the techniques of very exceptional divisors discussed in [1, Section 3]). This means that $S^{\prime} \cap F^{\prime}$ contains a $\log$ canonical center of $\left(X^{\prime}, \Delta^{\prime}+\right.$ $\left.b_{P}\left(f^{\prime}\right)^{*} P\right)$, where $S^{\prime}=\phi_{*} S$ as usual. Hence there exists a prime divisor $E$ over $S^{\prime}$ such that $a\left(E, S^{\prime}, \Delta_{S^{\prime}}+b_{P}\left(\left.f^{\prime}\right|_{S^{\prime}}\right)^{*} P\right)=-1$ and $E$ maps to $P$, where $K_{S^{\prime}}+\Delta_{S^{\prime}}=\left.\left(K_{X^{\prime}}+\Delta^{\prime}\right)\right|_{S^{\prime}}$. By the construction of $\phi: X \rightarrow X^{\prime}$, we have $a\left(E, S, \Delta_{S}+\right.$
$\left.b_{P}\left(\left.f\right|_{S}\right)^{*} P\right)=-1$. We note that $\left(X, \Delta^{<1}+\right.$ $\left.\operatorname{Supp} \Delta^{\geq 1}\right)$ is divisorial $\log$ terminal. Hence $S \cap F$ always contains a log canonical center of $(X, \Delta+$ $\left.b_{P} f^{*} P\right)$.

Remark 3.2. In Lemma 3.1, we assume that $F$ contains no $\log$ canonical center of $(X, \Delta+$ $\left.b_{P} f^{*} P\right)$. Then $\left(X, \Delta+\left(b_{P}+\varepsilon\right) f^{*} P\right)$ is $\log$ canonical in a neighborhood of $F$ for $0<\varepsilon \ll 1$. In this situation, $\left(S^{\nu}, \Delta_{S^{\nu}}+\left(b_{P}+\varepsilon\right) \nu^{*}\left(\left.f\right|_{S}\right)^{*} P\right)$ is log canonical by adjunction. Hence $\left(S^{\nu}, \Delta_{S^{\nu}}+b_{P} \nu^{*}\left(\left.f\right|_{S}\right)^{*} P\right)$ has no $\log$ canonical center mapping to $P$.

Lemma 3.3. Let $X$ be a normal quasi-projective variety and let $W$ be a closed subvariety of $X$. Let $\varphi: \widetilde{W} \rightarrow W$ be a projective birational morphism from a normal variety $\widetilde{W}$. Then we can construct a projective birational morphism $\psi: \widetilde{X} \rightarrow X$ from a normal variety $\widetilde{X}$ such that $\psi$ is an isomorphism over the generic point of $W$ with the following commutative diagram:

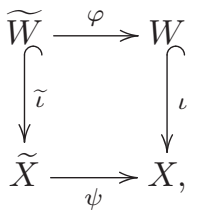

where $\iota$ and $\widetilde{\iota}$ are closed embeddings.

Proof. By [11, Chapter II, Theorem 7.17], there exists a coherent ideal sheaf $\mathcal{I}$ on $W$ such that $\varphi: \widetilde{W} \rightarrow W$ corresponds to the blow-up of $\mathcal{I}$. We put $\mathcal{J}=\operatorname{Ker}\left(\mathcal{O}_{X} \rightarrow \mathcal{O}_{W} \rightarrow \mathcal{O}_{W} / \mathcal{I}\right)$. Then $\mathcal{J}$ is a coherent ideal sheaf on $X$. Let $\psi^{\prime}: X^{\prime} \rightarrow X$ be the blow-up of $\mathcal{J}$. Then we obtain the following commutative diagram by $[11$, Chapter II, Corollary 7.15$]$

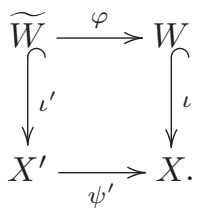

By construction, $\psi^{\prime}$ is an isomorphism over the generic point of $W$. Let $\nu: \widetilde{X} \rightarrow X^{\prime}$ be the normalization of $X^{\prime}$. Since $\widetilde{W}$ is normal by assumption, $\widetilde{W} \rightarrow X^{\prime}$ factors through $\widetilde{X}$. Then we get the desired diagram (3.1) such that $\widetilde{W} \rightarrow \widetilde{X}$ is a closed embedding and that $\psi: \widetilde{X} \rightarrow X$ is an isomorphism over the generic point of $W$.

Lemma 3.4. Let $\left(Y, \Delta_{Y}\right)$ be a Q-factorial divisorial log terminal pair and let $f: Y \rightarrow X$ be a projective morphism with $f_{*} \mathcal{O}_{Y} \simeq \mathcal{O}_{X}$ and $K_{Y}+$ 
$\Delta_{Y} \sim_{\mathbf{R}, f} 0$. Let $V$ be a reduced divisor on $Y$ such that $V \leq \Delta_{Y}^{=1}$ and $f\left(V_{i}\right)$ is independent of $i \in I$, where $V=\sum_{i \in I} V_{i}$ is the irreducible decomposition of $V$. $W e$ set $W=f\left(V_{i}\right)$. Suppose that $W \subsetneq X$ and that no log canonical center of $\left(Y, \Delta_{Y}-V\right)$ maps into $W$ by f. Then $f_{*} \mathcal{O}_{V} \simeq \mathcal{O}_{W}$ holds.

Proof. We can take a projective birational morphism $g: Z \rightarrow Y$ from a smooth variety $Z$ such that $g$ is an isomorphism over the generic point of any $\log$ canonical center of $\left(Y, \Delta_{Y}\right)$ and that $\operatorname{Exc}(g) \cup \operatorname{Supp} g_{*}^{-1} \Delta_{Y}$ is a simple normal crossing divisor on $Z$. Then we can write $K_{Z}+\Delta_{Z}=$ $g^{*}\left(K_{Y}+\Delta_{Y}\right)+E$ with $\Delta_{Z}=g_{*}^{-1} \Delta_{Y}$ such that $\lceil E\rceil$ is effective and $g$-exceptional. Let $V_{Z}$ be the strict transform of $V$ on $Z$. We have $-V_{Z}+$ $\lceil E\rceil \sim_{\mathbf{R}, f \circ g} K_{Z}+\Delta_{Z}-V_{Z}+\{-E\}$. By the vanishing theorem of Reid-Fukuda type (see [6, Theorem 3.2.11]), $R^{i} g_{*} \mathcal{O}_{Z}\left(-V_{Z}+\lceil E\rceil\right)=0$ for every $i>0$. We note that $g_{*} \mathcal{O}_{Z}\left(-V_{Z}+\lceil E\rceil\right) \simeq \mathcal{O}_{Y}(-V)$ holds since $\lceil E\rceil$ is effective and $g$-exceptional. We consider the following long exact sequence:

$$
\begin{aligned}
0 \longrightarrow f_{*} \mathcal{O}_{Y}(-V) & \longrightarrow f_{*} \mathcal{O}_{Y} \longrightarrow f_{*} \mathcal{O}_{V} \\
& \stackrel{\delta}{\longrightarrow} R^{1} f_{*} \mathcal{O}_{Y}(-V) \longrightarrow \cdots
\end{aligned}
$$

By $[5$, Theorem 6.3 (i) $]$ (see also $[6$, Theorem 3.16 .3 (i)]), there exists no associated prime of $R^{1} f_{*} \mathcal{O}_{Y}(-V) \simeq R^{1}(f \circ g)_{*} \mathcal{O}_{Z}\left(-V_{Z}+\lceil E\rceil\right)$ in $W=$ $f(V)$. Hence the above connecting homomorphism $\delta$ is zero. Therefore, $\mathcal{O}_{X} \simeq f_{*} \mathcal{O}_{Y} \rightarrow f_{*} \mathcal{O}_{V}$ is surjective. Thus the natural map $\mathcal{O}_{W} \rightarrow f_{*} \mathcal{O}_{V}$ is an isomorphism.

Let us start the proof of Theorem 1.2.

Proof of Theorem 1.2. We will prove $\mathbf{B}=$ $\mathbf{B}(W ; X, \Delta)$ under a slightly weaker assumption that $\Delta$ is only effective in a neighborhood of the generic point of $W$.

Step 1. We take an arbitrary projective birational morphism $\tilde{Z} \rightarrow W$ from a normal variety $\tilde{Z}$. We have to prove $\mathbf{B}_{\tilde{Z}}=\mathbf{B}(W ; X, \Delta)_{\tilde{Z}}$. By taking an affine open cover of $X$, we may assume that $X$ is quasi-projective. By applying Lemma 3.3 to $\tilde{Z} \rightarrow W$ and $W \hookrightarrow X$, we get the following commutative diagram:

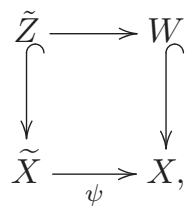

where $\psi: \widetilde{X} \rightarrow X$ is a projective birational morphism from a normal variety such that $\psi$ is an isomorphism over the generic point of $W$. We put $K_{\widetilde{X}}+\widetilde{\Delta}=\psi^{*}\left(K_{X}+\Delta\right)$. Since $\psi$ is an isomorphism over the generic point of $W, \widetilde{\Delta}$ is effective in a neighborhood of the generic point of $\tilde{Z}$ and $\tilde{Z}$ is a $\log$ canonical center of $(\widetilde{X}, \widetilde{\Delta})$. By replacing $(X, \Delta)$ and $W$ with $(\widetilde{X}, \widetilde{\Delta})$ and $\tilde{Z}$, respectively, we may further assume that $W$ is normal. By this reduction, all we have to do is to prove $\mathbf{B}_{W}=\mathbf{B}(W ; X, \Delta)_{W}$.

Step 2. We take an effective Cartier divisor $D$ on $X$ such that $W \not \subset \operatorname{Supp} D$ and $\operatorname{Supp} \Delta^{<0} \subset$ Supp $D$. We consider the pair $(X, \Delta+m D)$ for some sufficiently large positive integer $m$ such that $\Delta+m D$ is effective. We take a projective birational morphism $f: Y \rightarrow X$ from a smooth quasi-projective variety $Y$ such that $f^{-1}(W)$ and $\operatorname{Exc}(f)$ are divisors on $Y$ such that the union of $f^{-1}(W), \operatorname{Exc}(f)$, $\operatorname{Supp} f_{*}^{-1} \Delta$, and $\operatorname{Supp} f_{*}^{-1} D$ is contained in a simple normal crossing divisor. We put $K_{Y}+\Delta_{Y}=$ $f^{*}\left(K_{X}+\Delta\right)$ and $K_{Y}+\Delta_{Y}+m f^{*} D=f^{*}\left(K_{X}+\Delta+\right.$ $m D$ ). We define $V=\sum_{i \in I} V_{i}$, where $V_{i}$ runs over components of $\Delta_{Y}^{=1}$ with $f\left(V_{i}\right)=W$. By construction, there exists no $\log$ canonical center of $\left(Y, \Delta_{Y}-V\right)$ mapping to $W$ over a neighborhood of the generic point of $W$. Let $T$ be the prime divisor over $X$ which was chosen in order to define $\mathbf{B}(W ; X, \Delta)$. Let $S$ be any prime divisor over $X$ such that $a(S, X, \Delta)=-1$ and that the center of $S$ on $X$ is $W$. We may assume that $S$ and $T$ are components of $V$ by taking $f: Y \rightarrow X$ suitably. To prove Theorem 1.2, it is sufficient to check that $\alpha_{P, V_{i}}$ is independent of the choice of $i \in I$.

Step 3. By running a minimal model program with scaling of an ample divisor as in the proof of [7, Theorem 3.9], we get a dlt blow-up $f^{\prime}: Y^{\prime} \rightarrow X$ of $(X, \Delta+m D)$ with the following commutative diagram

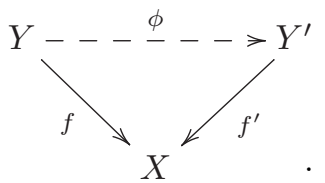

We put $K_{Y^{\prime}}+\Delta_{Y^{\prime}}=\left(f^{\prime}\right)^{*}\left(K_{X}+\Delta\right)$, and we define $\Gamma$ to be the sum of $\left(\Delta_{Y^{\prime}}+\left(f^{\prime}\right)^{*} m D\right)^{<1}$ and $\operatorname{Supp}\left(\Delta_{Y^{\prime}}+\left(f^{\prime}\right)^{*} m D\right)^{\geq 1}$. By construction, $Y^{\prime}$ is Q-factorial and $\left(Y^{\prime}, \Gamma\right)$ is divisorial $\log$ terminal. For the details, see [7, Theorem 3.9]. Therefore, $f^{\prime}:\left(Y^{\prime}, \Delta_{Y^{\prime}}\right) \rightarrow(X, \Delta)$ is a dlt blow-up over a neighborhood of the generic point of $W$. By construction again, $\phi$ does not contract any compo- 
nents of $V$. We put $V^{\prime}=\phi_{*} V$ and $V_{i}^{\prime}=\phi_{*} V_{i}$ for every $i \in I$. We note that $\left(Y^{\prime}, V^{\prime}\right)$ is divisorial $\log$ terminal since $V^{\prime} \leq \operatorname{Supp}\left(\Delta_{Y^{\prime}}+\left(f^{\prime}\right)^{*} m D\right)^{\geq 1}$. In particular, $V_{i}^{\prime}$ is normal for every $i \in I$. We can take a Zariski open neighborhood $U$ of the generic point of $W$ over which $f^{\prime}:\left(Y^{\prime}, \Delta_{Y^{\prime}}\right) \rightarrow(X, \Delta)$ is a dlt blowup and no $\log$ canonical center of $\left(Y^{\prime}, \Delta_{Y^{\prime}}-V^{\prime}\right)$ maps to $W$ by $f^{\prime}$. By applying Lemma 3.4 to $f^{\prime}:\left.\left(Y^{\prime}, \Delta_{Y^{\prime}}\right)\right|_{\left(f^{\prime}\right)^{-1}(U)} \rightarrow U$, we obtain that the natural map $\mathcal{O}_{W} \rightarrow f_{*}^{\prime} \mathcal{O}_{V^{\prime}}$ is an isomorphism on $U$. On the other hand, since every irreducible component of $V^{\prime}$ is dominant onto $W$, we see that $\operatorname{Spec}_{W} f_{*}^{\prime} \mathcal{O}_{V^{\prime}}$ is a variety. Therefore, since $W$ is normal, the finite birational morphism $\operatorname{Spec}_{W} f_{*}^{\prime} \mathcal{O}_{V^{\prime}} \rightarrow W$ is an isomorphism by Zariski's main theorem. This implies that $f^{\prime}: V^{\prime} \rightarrow W$ has connected fibers. We put $K_{V_{i}^{\prime}}+$ $\Delta_{V_{i}^{\prime}}=\left.\left(K_{Y^{\prime}}+\Delta_{Y^{\prime}}\right)\right|_{V_{i}^{\prime}}$ and $f_{i}^{\prime}=\left.f^{\prime}\right|_{V_{i}^{\prime}}: V_{i}^{\prime} \rightarrow W$ for every $i \in I$. We shrink $W$ and assume that $P$ is Cartier. Then we set

$\alpha_{P, V_{i}^{\prime}}=\sup \left\{\begin{array}{l|l}\lambda \in \mathbf{R} & \begin{array}{l}\left(V_{i}^{\prime}, \Delta_{V_{i}^{\prime}}+\lambda\left(f_{i}^{\prime}\right)^{*} P\right) \text { is } \\ \text { sub log canonical over } \\ \text { the generic point of } P\end{array}\end{array}\right\}$.

It is easy to see that $\alpha_{P, V_{i}}=\alpha_{P, V_{i}^{\prime}}$ holds for every $i \in I$. Therefore, to prove that $\alpha_{P, V_{i}}$ is independent of the choice of $i \in I$, it is sufficient to prove that $\alpha_{P, V_{i}^{\prime}}$ is independent of the choice of $i \in I$.

Step 4. We take a prime divisor $P$ on $W$. By cutting down $X$ by general hyperplanes, we assume that $W$ is a smooth curve and $P$ is a closed point. By shrinking $X$ suitably around $P,\left(V_{i}^{\prime}, \Delta_{V_{i}^{\prime}}\right)$ is divisorial $\log$ terminal over $W \backslash P$ for every $i \in I$. We put

$$
c_{P}=\sup \left\{\begin{array}{l|l}
\lambda \in \mathbf{R} & \begin{array}{l}
\left(V_{i}^{\prime}, \Delta_{V_{i}^{\prime}}+\lambda\left(f_{i}^{\prime}\right)^{*} P\right) \text { is } \\
\text { sub log canonical for } \\
\text { every } i \in I
\end{array}
\end{array}\right\} .
$$

By definition, there exists $i_{0} \in I$ such that $\alpha_{P, V_{i_{0}}^{\prime}}=$ $c_{P}$ holds. From now on, we will prove that $\alpha_{P, V_{i}^{\prime}}=$ $c_{P}$ holds for every $i \in I$. If $\# I=1$, then there is nothing to prove. Hence we may assume that $\# I \geq 2$. To obtain $\alpha_{P, V_{i}^{\prime}}=c_{P}$ for every $i \in I$, it is sufficient to prove the following claim.

Claim. Let $F$ be any connected component of $f_{i}^{\prime-1}(P)$ and let $B$ be any irreducible component of $\left(\Delta_{V_{i}^{\prime}}^{h}\right)=1$ for some $i \in I$. Then $B \cap F$ contains a log canonical center of $\left(V_{i}^{\prime}, \Delta_{V_{i}^{\prime}}+c_{P}\left(f_{i}^{\prime}\right)^{*} P\right)$.

Proof of Claim. We note that for any $j \in I$ there exists some $k \in I$ with $k \neq j$ such that $V_{j}^{\prime} \cap$ $V_{k}^{\prime} \neq \emptyset$ and that some irreducible component of $V_{j}^{\prime} \cap$ $V_{k}^{\prime}$ is dominant onto $W$ by $f^{\prime}$ since $f^{\prime}: V^{\prime} \rightarrow W$ has connected fibers and every irreducible component of $V^{\prime}$ is dominant onto $W$ by $f^{\prime}$. We take an irreducible component $A$ of $V_{j}^{\prime} \cap V_{k}^{\prime}$ with $j \neq k$ such that $A$ is dominant onto $W$ by $f^{\prime}$. We note that $A$ is an irreducible component of $\left(\Delta_{V_{j}^{\prime}}^{h}\right)^{=1}$ and $\left(\Delta_{V_{k}^{\prime}}^{h}\right)^{=1}$ by adjunction. Let $G_{j}$ be a connected component of $\left(f_{j}^{\prime}\right)^{-1}(P)$. Then $A \cap G_{j}$ contains a log canonical center of $\left(V_{j}^{\prime}, \Delta_{V_{j}^{\prime}}+c_{P}\left(f_{j}^{\prime}\right)^{*} P\right)$ if and only if $A \cap G_{k}$ contains a $\log$ canonical center of $\left(V_{k}^{\prime}, \Delta_{V_{k}^{\prime}}+\right.$ $\left.c_{P}\left(f_{k}^{\prime}\right)^{*} P\right)$, where $G_{k}$ is the connected component of $\left(f_{k}^{\prime}\right)^{-1}(P)$ containing $A \cap G_{j}$. We first apply Lemma 3.1 to $f_{i_{0}}^{\prime}:\left(V_{i_{0}}^{\prime}, \Delta_{V_{i_{0}}^{\prime}}+c_{P}\left(f_{i_{0}}^{\prime}\right)^{*} P\right) \rightarrow W$ and then use the connectedness of the fibers of $f^{\prime}: V^{\prime} \rightarrow$ $W$. By repeating this argument, we finally obtain that $B \cap F$ always contains a log canonical center of $\left(V_{i}^{\prime}, \Delta_{V_{i}^{\prime}}+c_{P}\left(f_{i}^{\prime}\right)^{*} P\right)$.

As we mentioned above, we see that $\alpha_{P, V_{i}^{\prime}}$ is independent of $i \in I$. This is what we wanted.

The above arguments show that $\mathbf{B}_{W}=\mathbf{B}(W$; $X, \Delta)_{W}$ holds. We finish the proof of Theorem 1.2.

Remark 3.5. In Step 3 in the proof of Theorem 1.2 , we proved that $f^{\prime}: V^{\prime} \rightarrow W$ has connected fibers. Note that $W$ is normal by the reduction argument in Step 1 in the proof of Theorem 1.2. However, it is not clear whether $f^{\prime}: V_{i}^{\prime} \rightarrow W$ has connected fibers or not. Hence we need a somewhat artificial formulation in Lemma 3.1.

Acknowledgments. The first author was partially supported by JSPS KAKENHI Grant Numbers JP19H01787, JP20H00111, JP21H00974. The second author was partially supported by JSPS KAKENHI Grant Numbers JP16J05875, JP19J00046.

\section{References}

[ 1 ] C. Birkar, Existence of log canonical flips and a special LMMP, Publ. Math. Inst. Hautes Études Sci. 115 (2012), 325-368.

[ 2 ] C. Birkar, P. Cascini, C. D. Hacon and J. McKernan, Existence of minimal models for varieties of log general type, J. Amer. Math. Soc. 23 (2010), no. 2, 405-468.

[ 3 ] S. Filipazzi, On a generalized canonical bundle formula and generalized adjunction, arXiv: 1807.04847.

[ 4 ] O. Fujino, Semi-stable minimal model program for varieties with trivial canonical divisor, Proc. Japan Acad. Ser. A Math. Sci. 87 (2011), no. 3, $25-30$.

[5] O. Fujino, Fundamental theorems for the log minimal model program, Publ. Res. Inst. Math. Sci. 47 (2011), no. 3, 727-789. 
[6] O. Fujino, Foundations of the minimal model program, MSJ Memoirs, 35, Mathematical Society of Japan, Tokyo, 2017.

[ 7 ] O. Fujino, Cone theorem and Mori hyperbolicity, arXiv:2102.11986.

[ 8 ] O. Fujino and K. Hashizume, Existence of $\log$ canonical modifications and its applications, arXiv:2103.01417.
[ 9 ] O. Fujino and K. Hashizume, Adjunction and inversion of adjunction, arXiv:2105.14531.

[ 10 ] C. D. Hacon, On the log canonical inversion of adjunction, Proc. Edinb. Math. Soc. (2) 57 (2014), no. 1, 139-143.

[11] R. Hartshorne, Algebraic geometry, Graduate Texts in Mathematics, No. 52, Springer-Verlag, New York, 1977. 